\title{
Rehmet, Frank, Neelke Wagner, Tim Willy Weber. 2020. Volksabstimmungen in Europa - Regelungen und Praxis im internationalen Vergleich
}

\author{
Opladen: Barbara Budrich, 202 Seiten, 26,80 $€$, ISBN \\ 978-3-8474-2275-4 (Paperback), eISBN 978-3-8474-1350-9 \\ (eBook)
}

\section{Hermann Heußner}

Online publiziert: 24. Juni 2020

(C) Der/die Autor(en) 2020

Volksabstimmungen prägen die Demokratie zunehmend und auf vielfältige Weise. Dies gilt insbesondere für Europa. Zuletzt konnte man sich dazu konzentriert in dem von Bruno Kaufmann und Dane Waters auf Englisch herausgegebenen Werk „Direct Democracy in Europe“ aus dem Jahr 2004 umfassend informieren. Es besteht also ein dringender Bedarf für ein aktuelles Nachschlagewerk zu Volksabstimmungen in Europa. In diese Lücke stößt das vorliegende, auf Deutsch verfasste „Überblickswerk".

Die Autor*innen betrachten die nationale Ebene von 43 Staaten. Die Türkei und Russland wollen sie schon deshalb nicht behandeln, weil sie nur zum Teil in Europa liegen, Weißrussland und den Vatikan sparen sie aus, weil sie keine Demokratien sind. Die Untersuchung umfasst - je nach Staat - den Zeitraum seit Einführung der Reglungen bis Ende 2018. Es werden nur die bis heute durchgehend demokratischen Zeiträume betrachtet. Dies hat zur Folge, dass der Betrachtungszeitraum der ehemals kommunistischen Staaten erst mit der Unabhängigkeit nach 1989 beginnt.

Das Ziel des Buches besteht darin, ,... Begriffe so zu definieren, dass sich mit ihnen die wesentlichen Varianten von Volksabstimmungen klar bestimmen lassen, Daten zu Volksabstimmungen und Volksbefragungen in den europäischen Staaten verständlich und übersichtlich darzustellen, die Verfahren und die Praxis in den jeweiligen Ländern zu bewerten ..." (S. 7).

Die Verfasser*innen unterscheiden zwischen verbindlichen Volksentscheiden und unverbindlichen Volksbefragungen. Volksentscheide differenzieren sie danach, wer sie auslöst: Bürger, Staatsorgane, Kombinationen aus beiden; dazu kommen obligatorische Volksentscheide. Zu den direktdemokratischen Verfahren zählen nur die von

Prof. Dr. H. Heußner $(\varangle)$

Öffentliches Recht und Recht der Sozialen Arbeit, Hochschule Osnabrück,

Breitscheidstr. 51, 34119 Kassel, Deutschland

E-Mail: h.heussner@hs-osnabrueck.de 
den Bürgern ausgelösten Volksentscheide, nämlich die Volksinitiative und das fakultative Gesetzesreferendum, sowie das obligatorische Referendum. Von Staatsorganen ausgelöste Entscheide zählen zu Recht nicht dazu, weil sie Manipulations- und/oder Plebiszitrisiken bergen. Die Autor*innen verwenden den Begriff Plebiszit für „,von oben“ ausgelöste Entscheide nicht. Für eine prägnante Begriffsbildung wäre dies jedoch zu empfehlen. So ist diese Bezeichnung auch in der Schweiz, dem europäischen Mutterland der direkten Demokratie, für von oben ausgelöste Abstimmungen als abgrenzender Begriff üblich, und er entspricht der historischen Begrifflichkeit, wonach im antiken Rom das ,plebiscitum“ nur als Antwort des römischen Volkes auf Vorlagen der Versammlungsleitung ,,von oben“ möglich war.

Um die Regelungen und Praxis der Volksabstimmungen darzustellen, leiten die Autor*innen aus den verschiedenen Verfahrenstypen und deren Anwendungshäufigkeit sieben Ländergruppen ab:

- Direktdemokratische Verfahren als Routineverfahren

- Direktdemokratische Verfahren mit einiger Praxis

- Direktdemokratische Verfahren mit wenig Praxis

- Direktdemokratische Verfahren als Ausnahmeverfahren - Volksabstimmungen durch Staatsorgane überwiegen

- Keine direktdemokratischen Verfahren, jedoch Volksabstimmungen durch Staatsorgane

- Nur unverbindliche Volksbefragungen

- Weder Volksabstimmungen noch Volksbefragungen

Diese Gruppenbildung spannt ein Spektrum auf, das einen guten Überblick verschafft. Am ,oberen“ Ende der Skala rangieren zwei Staaten: Die Schweiz und das sehr kleine Liechtenstein. Am „unteren“ Ende befinden sich Deutschland und Monaco. Deutschland, der größte Staat der untersuchten Länder, spielt demnach mit dieser Abstinenz eine absolute Sonderrolle. Diese wird freilich dadurch relativiert, dass alle Bundesländer Volksgesetzgebung aufweisen.

In der zweiten Gruppe ,von unten“ befinden sich sechs Staaten, die lediglich unverbindliche Volksbefragungen zulassen. Insgesamt kam es zu 14 Befragungen. Unter diesen Staaten befindet sich auch Großbritannien. Dort fand 2016 die Brexit-Volksbefragung statt. Dieser Urnengang dominiert seitdem den Diskurs über direkte Demokratie, obwohl es sich dabei um keine direktdemokratische Volksabstimmung handelte, sondern um ein Plebiszit ,von oben“. In der Öffentlichkeit ist dies meistens nicht hinreichend bekannt. Die negativen Merkmale unverbindlicher Plebiszite werden deshalb unreflektiert auf direktdemokratische Verfahren übertragen und diskreditieren diese zu Unrecht. Hier empfiehlt sich der aufklärende Blick in das vorliegende Buch. Er zeigt: Von Staatsorganen ausgelöste Befragungen sind besonders anfällig für machtpolitische Überlegungen. So wurde die Fragestellung des Brexit-Plebiszits der Komplexität des Themas nicht gerecht. Kompromissbildungen waren nicht möglich und der Willensbildungsprozess war viel zu kurz. Die Verfasser*innen betonen, dass ,zwei Befragungen mit anderen Fragestellungen, ein - die Befragung(en) vorbereitender - Bürgerrat wie in Irland, der partizipatorische und direkte Demokratie kombiniert, oder direktdemokratische Verfahren (...) wahrscheinlich zu besseren Ergebnissen geführt (hätten).“ (S. 167). Zu ergänzen wäre noch: 
Gäbe es in Großbritannien die Volksinitiative, hätte es nach 2016 aller Wahrscheinlichkeit nach einen von den Brexit-Gegnern angestoßenen erneuten Volksentscheid gegeben, in dem die Bürger*innen ihr Votum von 2016 hätten korrigieren können.

In den drei mittleren Ländergruppen befinden sich insgesamt 24 Staaten mit zusammen 68 Volksabstimmungen. Soweit es hier direktdemokratische Verfahren „von unten“ gibt (Albanien, Bulgarien, Kroatien, Malta, Nordmazedonien, Serbien, Luxemburg) finden diese jedoch nur selten statt, weil die Hürden für die Volksinitiative bzw. das fakultative Referendum meist hoch bis sehr hoch sind (kurze Sammelfristen, Beteiligungsquoren). Die meisten Abstimmungen sind obligatorische Referenden oder Plebiszite ,von oben“ durch Staatsorgane.

Die Masse der insgesamt 1050 Volksabstimmungen findet mit zusammen 985 Abstimmungen in den ersten beiden Ländergruppen statt. Davon entfallen allein $61 \%$ auf die Schweiz. Dies liegt u.a. an der sehr langen Praxis dort seit 1848 und den sehr niederschwelligen Reglungen für direktdemokratische Verfahren. So sind fast alle Themen zulässig, die Qualifikationsquoren mit ca. 1\% (fakultatives Referendum) bzw. ca. $2 \%$ (Volksinitiative) sehr niedrig, die Sammelfristen mit 100 Tagen bzw. 18 Monaten lang. Es gibt keine Abstimmungsquoren. Jeder Bürger erhält eine offizielle Abstimmungsbroschüre mit ausführlichen Informationen. Obligatorische Referenden sind zu 75, fakultative Referenden zu 43 und Volksinitiativen zu ca. $10 \%$ erfolgreich. Die beiden letzteren Instrumente haben zusätzlich starke indirekte Wirkungen.

Zur Recht führen die Autor*innen die vielfältigen Funktionen und Wirkungen an, welche die direktdemokratischen Verfahren in der Schweiz haben. Sie sind u.a. Problem-Seismografen, rückkoppeln die Abgeordneten an die Bürger, befrieden und entschärfen Konflikte, führen zu Kompromissen, fördern Partizipation, konfrontieren Menschen mit Themen und Sichtweisen außerhalb ihrer eigenen Lebenswelt, können Parlamentsgesetze bremsen und Innovationen beschleunigen, oder bringen neue Themen auf die Agenda. Die Schwächung des Parlaments soll hinnehmbar sein, weil über $90 \%$ der Gesetze ohne Referendum verabschiedet werden. Zwar sei der direktdemokratische Prozess langsamer, dafür aber auch gründlicher. Volksinitiativen führten etwas stärker zur Einschränkung von Minderheitenrechten.

Die Autor*innen ziehen ein sehr positives Fazit: die Schweiz sei in mehrfacher Hinsicht ein Vorzeigeland direktdemokratischer Verfahren. Dies liege u.a. an den anwendungsfreundlichen niedrigen Hürden. Die langen Sammelfristen würden der öffentlichen Diskussion Raum geben, das Fehlen von Abstimmungsquoren verhindere Boykotte, bei Gegenvorlagen des Parlaments sei ein doppeltes „Ja“ mit Stichfrage möglich. Die Medien berichteten ausführlich, es bestünden zahlreiche Orientierungsangebote, so auch Abstimmungsparolen der Parteien und Verbände. Insgesamt führe direkte Demokratie zu hohem Respekt vor Abstimmungsmehrheiten und Zufriedenheit mit dem Funktionieren der Demokratie.

Zu Recht zählt das Werk aber auch Schwächen der schweizerischen halb-direkten Demokratie auf. Es gibt nämlich keine verfassungsgerichtliche Kontrolle, gegen Völkerrecht verstoßende Initiativen gelangen zur Abstimmung und finanzielles Übergewicht einer Seite muss für die schwächere Seite unfair wirken. Zudem gibt es in der Schweiz keine Transparenzvorschriften, welche die Offenlegung finanzieller Quellen in Abstimmungskämpfen verlangen. Die Abstimmungsbeteiligung von 
durchschnittlich ca. $45 \%$ bewerten die Autor*innen nicht als zu niedrig. Denn in einer additiven Betrachtung beteiligen sich zwischen zwei Wahlen ca. $80 \%$ aller Bürger*innen mindestens einmal an einer Volksabstimmung.

In der zweiten Ländergruppe befinden sich neun Staaten, die direktdemokratische Verfahren mit einiger Praxis aufweisen (Italien, Irland, San Marino, Litauen, Dänemark, Slowenien, Slowakei, Ungarn, Lettland). Hier fanden 198 direktdemokratische Volksentscheide und 39 Plebiszite und sonstige Verfahren statt. Italien und Irland stechen mit 65 bzw. 41 direktdemokratischen Verfahren heraus.

In Italien gibt es im Wesentlichen das abrogative (aufhebende) Gesetzesreferendum, das seit 194863 Mal stattgefunden hat. Das Unterschriftenquorum ist mit 500.000 Unterschriften sehr niedrig. Restriktiv ist jedoch das Beteiligungsquorum von $50 \%$, das zu Boykottaufrufen der Gegner führt, so dass 38,5\% der Referenden „unecht“ scheiterten. Dennoch konnten sich immerhin 35,4\% der Anliegen durchsetzen. Dazu zählen z.B. die Referenden gegen die „Lex Berlusconi“ 2011. Die Volksinitiative gibt es nicht. Volksabstimmungen in Italien erfüllten z. B. die Funktion, dass kleinere Parteien gesellschaftliche Debatten anstoßen konnten und richtungsweisende Entscheidungen gesellschaftlich brennender Fragen entschieden wurden (z. B. Ende der Atomkraft). Wenn Politiker ihr politisches Schicksal mit dem Erfolg in Volksabstimmungen verbinden, kann dies zu Rücktritten führen, so wie 2016 bei der Ablehnung der von Ministerpräsident Renzi propagierten Verfassungsreformen.

In Irland ist für Verfassungsänderungen ein obligatorisches Referendum notwendig, das seit 193741 Mal stattgefunden hat. Auf diese Weise wurde 2015 die gleichgeschlechtliche Ehe eingeführt und 2018 der Schwangerschaftsabbruch liberalisiert. Das Verfahren im Vorfeld der Abstimmung ist vorbildlich ausgestaltet. Zum einen gibt es seit 1998 die neutrale Referendumskommission, die abstimmungsrelevante Informationen für die Medien und zur Versendung an alle Haushalte erstellt. Zum anderen gibt es in den letzten Jahren Bürgerräte, deren Mitglieder per Los bestimmt werden. Sie erarbeiten Vorschläge für Verfassungsänderungen.

Ungarn ist von besonderem Interesse, weil dieses Land Zeichen einer autoritären Transformation aufweist. Hier fanden seit 198911 direktdemokratische Volksentscheide statt, 10 davon aufgrund der Volksinitiative. Seit 2008 hat es keinen ,von unten“ initiierten Volksentscheid mehr gegeben. Die Fidesz-Partei von Ministerpräsident Orban hat seit 2010 die Anforderungen an die Einreichung einer Initiative hochgeschraubt. Stattdessen hat die Fidesz-Partei 2016 ein Parlamentsplebiszit gegen das Recht der Europäischen Union, Quoten für Flüchtlinge festzulegen, durchgeführt. Dies ist der klassische Versuch, die Regierungspolitik durch das Volk ,,von oben“ legitimieren zu lassen.

Zur Qualitätsmessung dient den Autor*innen, die sich an einem partizipativen Demokratiebegriff orientieren, insbesondere der Umfang der Themenausschlüsse, die Anzahl der zu sammelnden Unterschriften, die Existenz von Abstimmungsquoren, die Länge von Fristen, die Form der Unterschriftensammlung, die Verteilung offizieller Abstimmungsbroschüren, die Interaktionsmöglichkeiten mit dem Parlament und die auf Fairness und Chancengleichheit ausgelegte Abstimmungskampfregulierung. Gemessen an diesen Kriterien weisen nur wenige Staaten weitgehend gute Regelungen auf: Im Wesentlichen die Schweiz, Liechtenstein und San Marino, mit 
Abstrichen Italien und Irland. So gibt es direktdemokratische Verfahren, bei denen die Bürger selbst initiativ werden können, auch nur in relativ wenigen Staaten: Die Volksinitiative in elf Ländern, neben der Schweiz und Liechtenstein im Wesentlichen nur in Staaten des ehemaligen Ostblocks. Das fakultative Referendum existiert nur in acht Ländern, neben der Schweiz und Liechtenstein vor allem in Italien. Die sehr ungleiche Anwendungshäufigkeit zwischen den Staaten zeigt, ,dass das bloße Vorhandensein direktdemokratischer Instrumente nicht ausreicht, um eine lebendige Praxis anzustoßen. Das ,Wie“ entscheidet.“(S. 40)

Die Autor*innen haben die Ziele ihres Buches gut eingelöst. Es ist ein hervorragendes, kompaktes Nachschlagewerk entstanden, welches das Zeug zum Standardwerk hat. Wichtig ist, dass es in relativ kurzen Abständen in neuen Auflagen erscheint, um aktuell zu bleiben. Jedem, der sich einen Überblick und Einstieg in die europäische Welt der Volksabstimmungen verschaffen will, sei es wärmstens empfohlen.

Funding Open Access funding provided by Projekt DEAL.

Open Access Dieser Artikel wird unter der Creative Commons Namensnennung 4.0 International Lizenz veröffentlicht, welche die Nutzung, Vervielfältigung, Bearbeitung, Verbreitung und Wiedergabe in jeglichem Medium und Format erlaubt, sofern Sie den/die ursprünglichen Autor(en) und die Quelle ordnungsgemäß nennen, einen Link zur Creative Commons Lizenz beifügen und angeben, ob Änderungen vorgenommen wurden.

Die in diesem Artikel enthaltenen Bilder und sonstiges Drittmaterial unterliegen ebenfalls der genannten Creative Commons Lizenz, sofern sich aus der Abbildungslegende nichts anderes ergibt. Sofern das betreffende Material nicht unter der genannten Creative Commons Lizenz steht und die betreffende Handlung nicht nach gesetzlichen Vorschriften erlaubt ist, ist für die oben aufgeführten Weiterverwendungen des Materials die Einwilligung des jeweiligen Rechteinhabers einzuholen.

Weitere Details zur Lizenz entnehmen Sie bitte der Lizenzinformation auf http://creativecommons.org/ licenses/by/4.0/deed.de.

\section{Literatur}

Kaufmann, Bruno, und Dane Waters (Hrsg.). 2004. Direct democracy in Europe. A comprehensive reference guide to the initiative and referendum process in Europe. Durham: Carolina Academic Press. 